\title{
Design and Simulation of RFID Aperture Coupled Fractal Antennas
}

\author{
Regular Paper
}

\author{
Dhrgham. K. Naji ${ }^{1}$, Jaber. S. Aziz ${ }^{2}$ and Raad S. Fyath,"* \\ 1,2,3 Department of Electronics and Communication Engineering, College of Engineering, Al-Nahrain University, Baghdad-Iraq \\ * Corresponding author E-mail: rsfyath@yahoo.com
}

Originally published in the International Journal of Radio Frequency Identification \& Wireless Sensor Networks, ISSN $1847-9812$

(C) 2012 Naji et al.; licensee InTech. This is an open access article distributed under the terms of the Creative Commons Attribution License (http://creativecommons.org/licenses/by/3.0), which permits unrestricted use, distribution, and reproduction in any medium, provided the original work is properly cited.

\begin{abstract}
Different fractal antennas based on aperture coupled feeding technique are studied, analysed and compared for size reduction, low profile, and good omini-dirctional pattern suitable for RFID applications. The results are used as a guideline to propose modified fractal shapes patch antennas having lower size reduction parameters. All the designed antennas are based on zero - order square patch antenna where different fractal geometries are introduced to miniaturize the size of antenna for $5.8 \mathrm{GHz}$ band. A maximum reduction in patch antenna size of $59.80 \%$ is achieved when using modified Minkowski pre-fractal patch antenna.
\end{abstract}

Keywords Aperture Coupled, Fractal Antenna, RFID

\section{Introduction}

Radio frequency Identification (RFID), which was developed around World War II, is a technology that provides wireless identification and tracking capability. In recent years, RFID technology has been rapidly developed and applied to many service industries, distribution logistics, manufacturing companies, goods flow systems, and mobile dongle applications [1, 2].
Several frequency bands have been assigned to RFID applications: $125 \mathrm{kHz}, 13.56 \mathrm{MHz}, 869 \mathrm{MHz}, 902-928$ $\mathrm{MHz}, 2.450$ (2.400-2.483) GHz and 5.800 (5.725-5.875) $\mathrm{GHz}$. The advantage of operating at higher frequencies is a longer range with high data transfer rate [3].

The rapid advancements in RFID technologies are the driving force in design and development of compact and light weight antennas [4, 5]. These features can be obtained using fractal geometry-based antennas [6, 7]. Fractals naturally arise in many mathematical fields which work with recursive procedures, for example, in dynamical systems and stochastic processes [8]. Applying fractals to the antenna elements allows for smaller size, multi-band and broad-band properties $[9,10]$.

Fractals have self-similar shapes and can be subdivided in parts such that each part is a reduced size copy of the whole. Self-similarity of fractals causes multi-band and broad-band properties and their complicated shapes provide design of antennas with smaller size. Fractals have convoluted and jagged shapes with many corners that these discontinuities increase bandwidth and the effective radiation of antennas. Fractals can be placed long electrical length in to a small area using their ability of space filling [11]. 
Fractal antennas have some very special properties [9, 10] that make them attractive for the design of small antennas. These properties are

- Broad band operation - the fractal antennas radiate and detect efficiently within a wide range of frequencies. The frequency range is specified by the smallest and largest size present in the antenna. The radiation pattern, and hence the detection efficiency do not vary much as a function of frequency.

- Gain - the fractal antennas can display a gain that depends relatively slowly on frequency over a large frequency range.

- Spatial structure - the fractal antennas can display a spatial structure which is also related to the antenna gain, as the antenna concentrate radiated power in certain positions and not in others.

Even though the theory behind fractal antennas is quite complex, their applicability to RFID is promising. In order to achieve the desired features of RFID antenna (compact size, low profile, environment insensitive, conformal and multi hand or broadband operation), a planar microstrip configuration with fractal geometry is one of the possibilities.

For microstrip patch antennas, aperture coupling [12, 13] is preferred to other feeding mechanisms as it offers greater design flexibility. Aperture coupling has considerable advantage as a feeding mechanism particularly in fractal designs where identifying a suitable feed position on such complex geometrical shapes is particularly difficult [14, 15]. In Ref. [16], a modified square fractal aperture coupled patch antenna has been used for ISM frequency band $2.4 \mathrm{GHz}-2.485 \mathrm{GHz}$ and from $5.725 \mathrm{GHz}$ to $5.875 \mathrm{GHz}$ for RFID.

This paper presents design issues related to miniaturized aperture coupled fractal antennas suitable for $5.8 \mathrm{GHz}$ ISM RFID band. The rest of the paper is organized as follows. Section II introduces the main concepts behind aperture coupled patch antenna. Section III presents various fractal patch antenna geometries along with design concepts. Modified fractal RFID patch antennas are given in section IV. Hybrid modified Koch fractal antennas are present in section V. Relation comparison of different types of fractal patch antennas and the main conclusions drawn from this study is presented in sections VI and VII, respectively. Simulation is presented using CST MWS 2010.

\section{Aperture Coupled Feeding}

The geometry of the aperture-coupled feeding is shown in Fig.1. This antenna consists of two substrates separated by a ground plane. The top substrate $\left(\varepsilon_{r 1}\right)$ contains the radiating element, and the bottom substrate $\left(\varepsilon_{r 2}\right)$ contains the microstrip feed line. A small aperture is cut in the ground plane to allow coupling from open circuited microstrip feed line to the radiating patch. The parameters on which resonant frequency of the antenna depends upon are shape and dimensions of the radiating element, dimensions of the ground plane, shape and size of the aperture, dimensions of feed line, position of feed line and the dielectric constants of the two substrates.

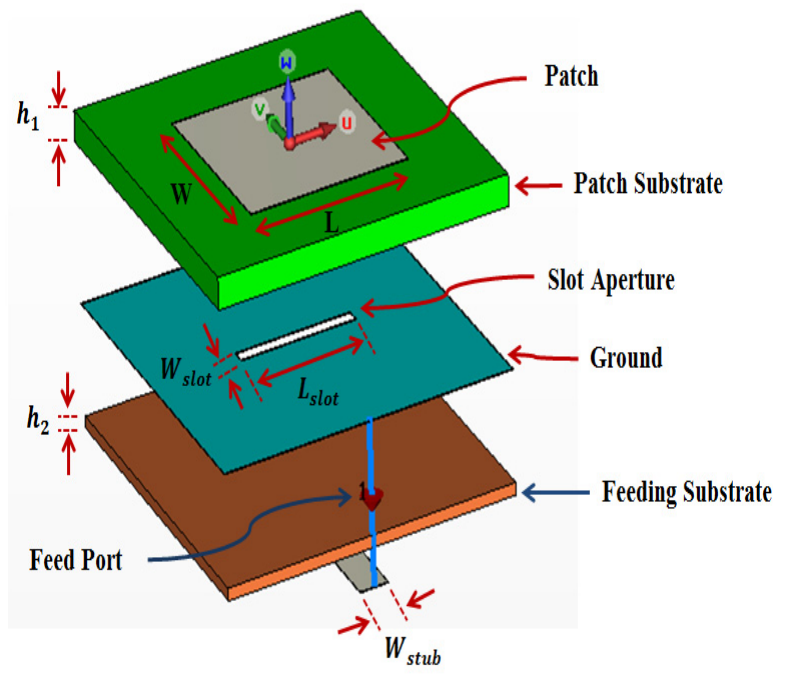

Figure 1. Aperture coupled patch antenna.

\section{Fractal Antenna Geometries and Design Concepts}

\subsection{Reference Patch}

A simple microstrip patch antenna is known as the iteration zero of fractal patch antenna. For iteration zero the basic formulas for determining the length and width of a microstrip patch antenna are used. These formulas are [17]

$$
\begin{gathered}
W=\frac{v_{o}}{2 f_{r}} \sqrt{\frac{2}{\varepsilon_{r}+1}} \\
\varepsilon_{e f f}=\frac{\varepsilon_{r}+1}{2}+\frac{\varepsilon_{r}-1}{2}\left(\frac{1}{\sqrt{1+12 h / W}}\right) \\
\Delta L=0.412 h \frac{\left(\varepsilon_{e f f}+0.300\right)\left[\frac{W}{h}+0.264\right]}{\left(\varepsilon_{e f f}-0.258\right)\left[\frac{W}{h}+0.813\right]} \\
L=\frac{v_{o}}{2 f_{r} \sqrt{\varepsilon_{e f f}}}-2 \Delta L
\end{gathered}
$$

where $W$ is the width, $L$ is the length, $\Delta L$ is the length reduced from the antenna to reduce fringing effects, $\varepsilon_{r}$ is the dielectric constant, $v_{o}$ is the speed of wave in free space, and $\varepsilon_{e f f}$ is the effective dielectric constant. Using Eqns. 1-4, the patch antenna is designed using the following parameters

$$
\varepsilon_{r 1}=4.3, h 1=1.6 \mathrm{~mm}, \quad \text { and } f_{r}=5.8 \mathrm{GHz}
$$


The dimensions of the patch are calculated to be

$$
L=11 \mathrm{~mm}, \quad W=14 \mathrm{~mm}
$$

The designed patch antenna is simulated using CST MWS 2010. The simulation takes into account various structure parameters associated with the coupled aperture such as slot length $L_{\text {slot }}$, slot width $W_{\text {slot }}$, and strip line parameters: strip length $L_{\text {strip }}$ and strip width $W_{\text {strip }}$. Finally substrate length $L_{s u b}$, and substrate width $W_{\text {sub }}$ parameters are used.

The simulation yields a resonance frequency of $6.4 \mathrm{GHz}$ which is higher than the target one $5.8 \mathrm{GHz}$. The patch structure parameters are then optimized to yield a simulated resonance frequency of $5.8 \mathrm{GHz}$ under square patch condition $L=W=10 \mathrm{~mm}$. These parameter values will be used later as the starting point (reference patch) to design various fractal antennas considered in this work. Table 1 shows the optimized antenna parameters for the reference patch antenna.

\begin{tabular}{cccc}
\hline Parameters & Values & Parameters & Values \\
\hline $\boldsymbol{L}=\boldsymbol{W}$ & 10 & $\boldsymbol{W}_{\text {strip }}$ & 2.4 \\
$\boldsymbol{L}_{\text {slot }}$ & 6.43 & $\boldsymbol{W}_{\text {slot }}$ & 0.5 \\
$\boldsymbol{L}_{\text {strip }}$ & 5 & $\boldsymbol{h}_{\mathbf{1}}$ & 1.6 \\
$\boldsymbol{L}_{\text {sub }}$ & 20 & $\boldsymbol{h}_{\mathbf{2}}$ & 0.8 \\
$\boldsymbol{W}_{\text {sub }}$ & 20 & $\boldsymbol{\varepsilon}_{\boldsymbol{r} \mathbf{1}}$ & 4.3 \\
& & $\boldsymbol{\varepsilon}_{\boldsymbol{r} \mathbf{2}}$ & 2.33
\end{tabular}

Table 1. Optimized antenna parameters in $(\mathrm{mm})$ to achieve $f_{r}=5.8 \mathrm{GHz}$ for reference square patch antenna.

Figure 2 shows simulation results related to the reflection return loss $S_{11}$ of the optimised reference patch antenna.

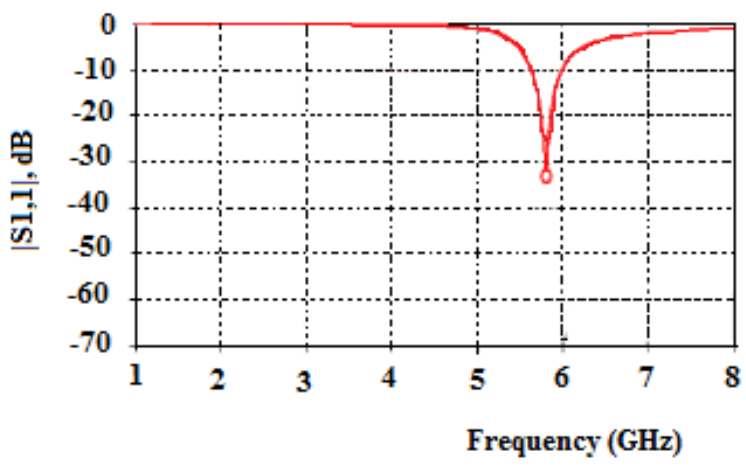

Figure 2. Simulation return loss $S_{11}$ of reference patch antenna. The 3D patterns as well the 2D copolar and crosspolar patterns for the reference patch are also introduced in Figure 3.

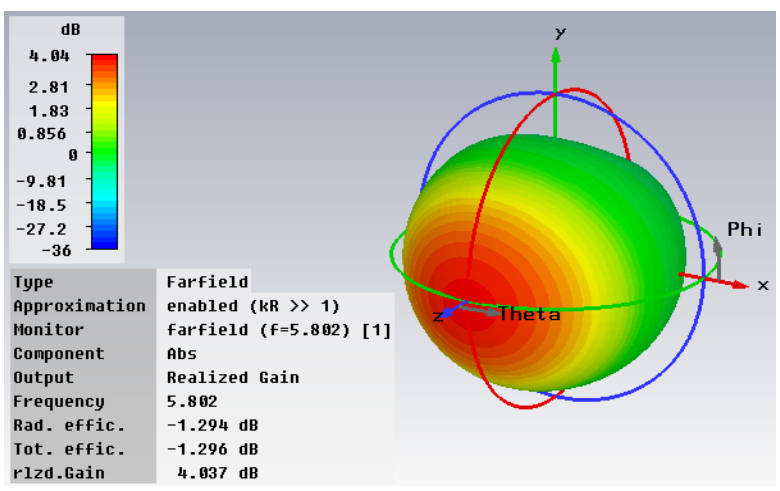

a. 3D pattern

Realized Gain Copolar (Phi=0)

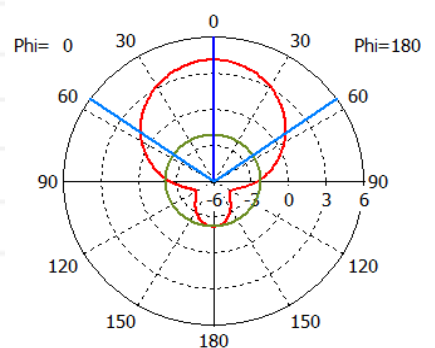

farfield $(f=5.802)[1]$ b. Copolar

Realized Gain Crosspolar (Phi $=0$ )

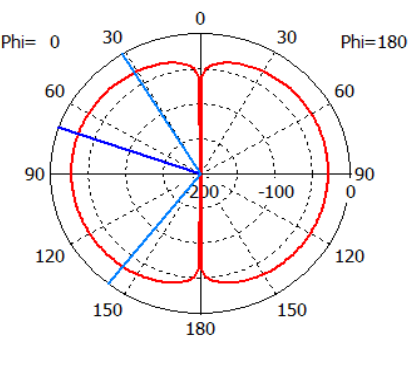

Theta / Degree vs. dB

c. Crosspolar
Frequency $=5.802$

Main lobe magnitude $=4.0 \mathrm{~dB}$ Main lobe direction $=0.0$ deg. Angular width $(3 \mathrm{~dB})=110.0 \mathrm{deg}$ Side lobe level $=-6.2 \mathrm{~dB}$

farfield $(f=5.802)[1]$

Frequency $=5.802$ Main lobe magnitude $=-29.2 \mathrm{~dB}$ Main lobe direction $=71.0$ deg. Angular width $(3 \mathrm{~dB})=110.4 \mathrm{deg}$.

Figure 3. Patterns of the reference patch.

It is worth to mention here that introducing fractal geometry on the reference patch antenna will reduce the resonant frequency. The dimension of the patch should be shortened as in Fig. 4 to produce $f_{r}=5.8 \mathrm{GHz}$.

The figure of merit used to measure the quality of the fractal antenna in this paper is the area reduction factor $\Delta \mathrm{A}$ defined as

$$
\Delta A=\left.\left(1-\frac{L_{f}^{2}}{L^{2}}\right)\right|_{f_{r}=5.8 \mathrm{GHz}}
$$

where $L$ and $L_{f}$ is the length of the square patch in the absence and presence of fractal geometry, respectively. 


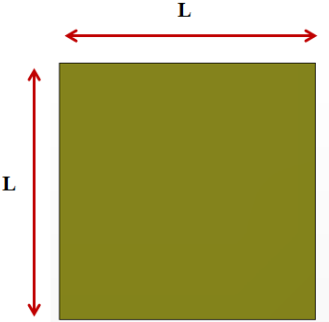

a. b.

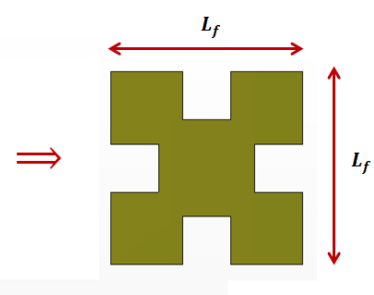

Figure 4. Area size reduction for fractal patch antenna $a$. Reference patch $b$. Fractal patch.

\section{2 . Sierpinski Carpet Fractal Patch Antenna}

Sierpinski carpet fractal antenna is the most widely studied fractal geometry for antenna application [18, 19]. The fractal antenna consists of geometrical shapes that are repeated; each ones have a unique attributes. The self-similarity that distributed on this antenna expected to cause its multi-band characteristic. On the others hand its can solve a traditional antenna that operate at single frequency.

The following formulas are used to add further iterations after designing the zero iteration of Sierpinski carpet geometry

$$
\begin{gathered}
N^{(n)}=8^{n} \\
A^{(n)}=(8 / 9)^{n} L_{o}^{2}
\end{gathered}
$$

Further one can show that

$$
L_{\text {perim }}{ }^{(n)}=4\left(1+\sum_{i=1}^{n} 8^{i-1} / 3^{i}\right) L_{o}
$$

where $L_{o}$ denotes the length of the reference patch, $N^{(n)}$ is the number of squares which will be covered with a radiating material, $L_{\text {perim }}{ }^{(n)}$ is the perimeter length at iteration $n$, and $A^{(n)}$ is the area of patch at iteration $n$.
As the iterations are added, some area of the radiating patch is removed and therefore, the physical dimensions of the radiating patch reduce. Thus the initial resonating frequency will shift from $5.8 \mathrm{GHz}$ to some lower frequency because of increasing the current path length. As the radiating patch continues to reduce in size, the resonating frequency will continue to shift to lower frequency bands as further iterations are added. This property helps in achieving higher multiple bands, while maintaining the original size of the patch.

Sierpinski carpet fractal patch antenna designed with one, two and three iterations are analysed and simulated and the results are listed in Table 2 . The results include antenna dimensions, scattering parameter $S_{11}$, bandwidth, and gain at $5.8 \mathrm{GHz}$. One can notice that when the iteration increases more reduction in patch size is obtained. With three iteration, a patch antenna size reduction of $20.79 \%$ is obtained. A new design concept is deduced from these results that more reduction is obtained when the ratio of perimeter length of patch to its enclosed area becomes higher.

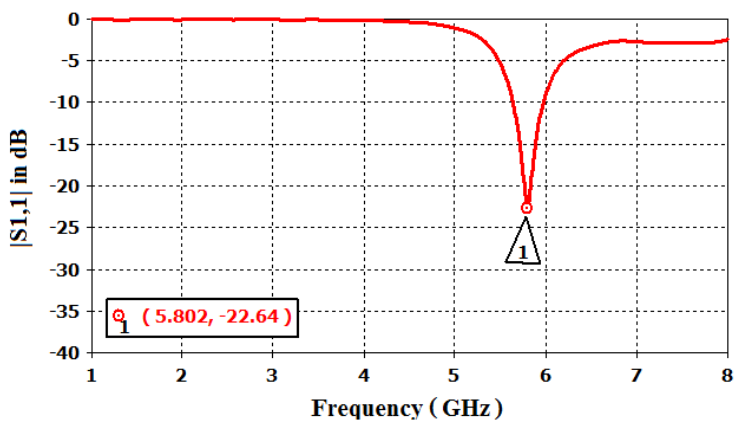

\begin{tabular}{|c|c|c|c|c|}
\hline & Zero Iteration & First Iteration & Second Iteration & Third Iteration \\
\hline$\Delta \boldsymbol{A}(\%)$ & - & 13.51 & 15.36 & 20.79 \\
\hline$L_{\text {perim }}(\mathrm{mm})$ & 40.00 & 49.60 & 69.51 & 112.00 \\
\hline$A\left(\mathrm{~mm}^{2}\right)$ & 100.00 & 76.88 & 66.87 & 55.63 \\
\hline$L_{\text {perim }} / A$ & 0.40 & 0.64 & 1.04 & 2.15 \\
\hline$S_{11}(d B)$ & -33.20 & -37.19 & -29.43 & -23.39 \\
\hline$B W(M H z)$ & 356 & 341 & 340 & 340 \\
\hline Gain (dB) & 4.04 & 3.80 & 3.78 & 3.66 \\
\hline
\end{tabular}

Figure 5. Simulation return loss $S_{11}$ of third iteration Sierpinski fractal patch antenna.

Table 2. Simulated parameters of Sierpinski carpet fractal antenna. 
Figure 5 shows the $S_{11}$ simulation of the third iteration Sierpinski fractal patch antenna. The 3D patterns as well the copolar and crosspolar patterns (yz plane) at $5.8 \mathrm{GHz}$ for the third iteration Sierpinski fractal patch antenna are introduced in Fig. 6.
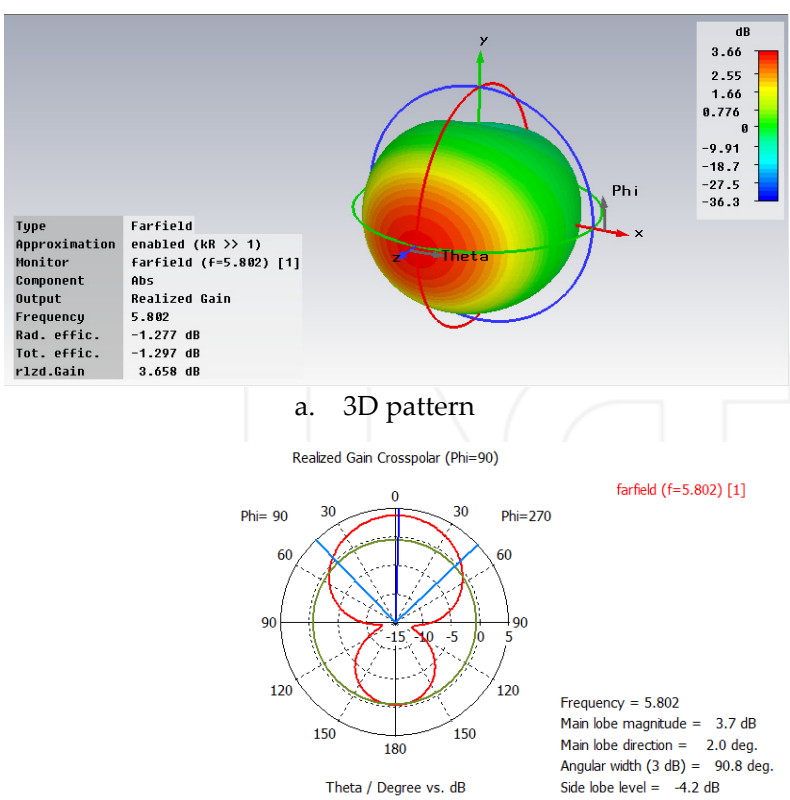

b. Copolar

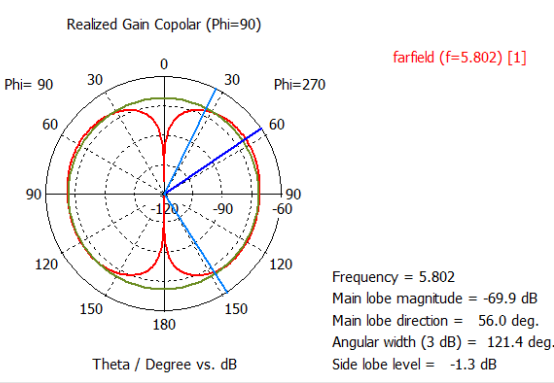

c. Crosspolar

Figure 6. Patterns of the third iteration Sierpinski fractal patch antenna.

\subsection{Minkowski Fractal Antenna}

The application of fractal geometry to conventional antenna structures optimizes the shape of the antennas in order to increase their electrical length, which thus reduces their overall size. Though different fractal geometries are available, a very few can be used in the design of microstrip antennas. One such geometry is Minkowski fractal antenna [20-22].

The fractal dimension $\mathrm{D}$ is a number which characterizes fractal structures. This parameter can be understood as a measurement of the space-filling ability by a fractal form. There are different definitions of D. One of them, which we use here, is the Hausdorff-Besicovich dimension (or self-similarity dimension) [11]. In this definition, the dimension $\mathrm{D}$ is the solution of the equation

$$
k_{1}\left(\frac{1}{h_{1}}\right)^{D}+k_{2}\left(\frac{1}{h_{2}}\right)^{D}+\cdots+k_{m}\left(\frac{1}{h_{m}}\right)^{D}=1
$$

where $k_{m}$ is the number of the copies of the initiator scaled by $h_{m}$ and $m$ is the number of different scale that the fractal possesses. For the Minkowski fractal antenna there are three scales $\left(1-a_{1}\right), a_{2}$, and $a_{1}$, and $k_{1}=2$, $k_{2}=2, k_{3}=1$, thus Eqn. 9 becomes

$$
2\left(\frac{1}{2}\left(1-a_{1}\right)\right)^{D}+2\left(a_{2}\right)^{D}+\left(a_{1}\right)^{D}=1
$$

where $a_{1}$ is the ratio $W_{1} / L_{o}$ and $a_{2}$ is the ratio $W_{2} / L_{o}$. Then according to Eqn. 9, when $a_{1}$ or $/$ and $a_{2}$ are varied, fractal structures with different dimensions may be obtained. The dimension of the traditional Minkowski island fractal, according to Eqn.10 is equal to 1.465 where in such case $a_{1}$ and $a_{2}$ both are equal to $1 / 3$ [Fig. 7].

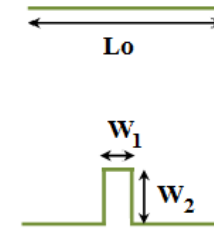

a.

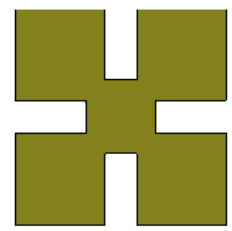

b.

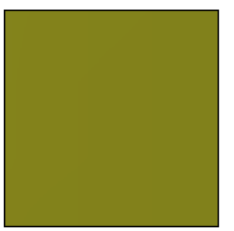

c.
Figure 7. Construction of the Minkowski-like fractal structure $a$. the generator $b$. first iteration, $c$. square patch antenna.

The design of fractal Minkowski patch antenna begins from the design of the zeroth iteration which is as the same as in Sierpinski Carpet antenna. The perimeter of one side antenna at the $\mathrm{n}$ iteration is found as

$$
L_{\text {perim }}^{(n)}=\left(1+2 a_{2}\right) L_{\text {perim }}^{(n-1)}
$$

where $L_{\text {perim }}{ }^{(n)}$ is the perimeter at iteration number $n$ and $L_{\text {perim }}{ }^{(n-1)}$ is the perimeter at iteration number $(n-1)$. The area of patch after first iteration is

$$
A^{(1)}=\left(1-4 a_{1} a_{2}\right) L_{o}^{2}
$$

and the area after second iteration is

$$
A^{(2)}=\left(1-2 a_{1} a_{2}\left(3+3 a_{1}^{2}-2 a_{1}+4 a_{2}^{2}\right)\right) L_{o}{ }^{2}
$$

It's found that the ratio of perimeter to area for any scales $a_{1}, a_{2}$ and at any iteration gives an indication on the reduction in patch antenna size. 
Two iterations of fractal Minkowski fractal antenna with different scales are simulated and the results are listed in Tables 3 and 4 . From these tables a large reduction in patch size $(\%)$ is obtained when $a_{1}=a_{2}=0.33$ which corresponds to a large ratio of perimeter length to enclosed area as indicated for Sierpinski carpet patch antenna. The return loss $S_{11}$ as well bandwidth and gain are also listed in Tables 3 to 4 .

The copolar and crosspolar patterns of the second iteration Minkowski fractal patch antennas when $a_{1}=a_{2}=0.33$ are introduced in Fig. 8. Figure 9 show the $S_{11}$ characteristics for the second iteration Minkowski fractal patch antenna when $b=0.33$ and 0.22 , respectively.

The results are displayed for three values of the scale parameter $a: 0.11,0.22$ and 0.33 . When the scale $a$ increases for a fixed values of the scale $b$, the resonance frequency deceases, since the perimeter length of the patch increases. When $b=0.33$, the resonance frequency is $6.880,6.285$, and $5.802 \mathrm{GHz}$ when $a=0.11,0.22$ and 0.33 respectively. These values are to be compared with , 5.802, and $5.704 \mathrm{GHz}$, respectively, when $b=0.22$

\begin{tabular}{|c|c|c|c|c|c|}
\hline$a_{1}, a_{2}$ &,-- & $0.33,0.22$ & $0.33,0.33$ & $0.11,0.11$ & $0.05,0.33$ \\
\hline$D$ & 1.000 & 1.298 & 1.465 & 1.172 & 1.549 \\
\hline$\Delta \boldsymbol{A}(\%)$ & - & 19.00 & 39.63 & 13.51 & 19.00 \\
\hline$L_{\text {perim }}(\mathrm{mm})$ & 40.00 & 52.00 & 51.80 & 45.46 & 44.00 \\
\hline$A\left(m m^{2}\right)$ & 100.00 & 57.00 & 33.54 & 82.21 & 79.00 \\
\hline$L_{\text {perim }} / A$ & 0.400 & 0.912 & 1.545 & 0.553 & 0.557 \\
\hline$S_{11}(d B)$ & -33.20 & -21.33 & -24.06 & -22.68 & -27.00 \\
\hline$B W(M H z)$ & 356 & 218 & 171 & 337 & 272 \\
\hline Gain (dB) & 4.04 & 3.43 & 2.56 & 3.80 & 3.58 \\
\hline
\end{tabular}

Table 3. Simulated parameters of the first iteration fractal Minkowski patch antenna.

\begin{tabular}{|c|c|c|c|c|c|c|}
\hline & & 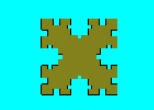 & & 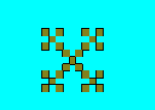 & 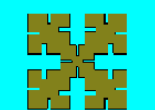 & 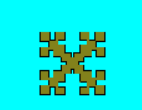 \\
\hline$a_{1}, a_{2}$ & 0,0 & $0.22,0.22$ & $0.33,0.22$ & $0.33,0.33$ & $0.11,0.33$ & $0.22,0.33$ \\
\hline$D$ & 1.000 & 1.306 & 1.298 & 1.465 & 1.509 & 1.471 \\
\hline$\Delta \boldsymbol{A}(\%)$ & - & 29.44 & 36.00 & 49.02 & 38.69 & 47.29 \\
\hline$L_{\text {perim }}(\mathrm{mm})$ & 40.00 & 70.10 & 66.76 & 72.34 & 87.00 & 80.67 \\
\hline$A\left(m^{2}\right)$ & 100.00 & 50.34 & 36.84 & 15.73 & 46.50 & 28.12 \\
\hline$L_{\text {perim }} / A$ & 0.400 & 1.392 & 1.812 & 5.042 & 1.870 & 2.868 \\
\hline$S_{11}(d B)$ & -33.20 & -42.10 & -51.30 & -49.21 & -43.15 & -47.64 \\
\hline$B W(M H z)$ & 356 & 245 & 239 & 156 & 239 & 192 \\
\hline Gain (dB) & 4.04 & 3.31 & 3.16 & 1.78 & 3.02 & 2.59 \\
\hline
\end{tabular}

Table 4. Simulated parameters of the second iteration fractal Minkowski patch antenna. 


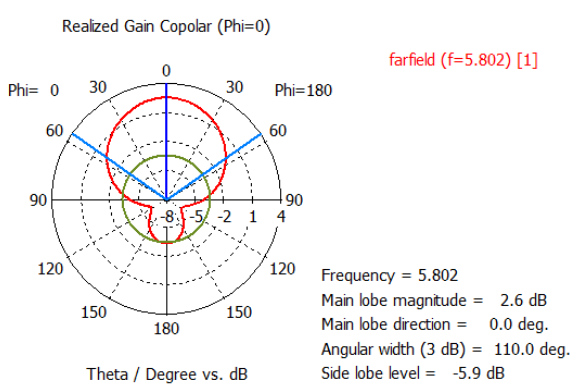

a. Copolar

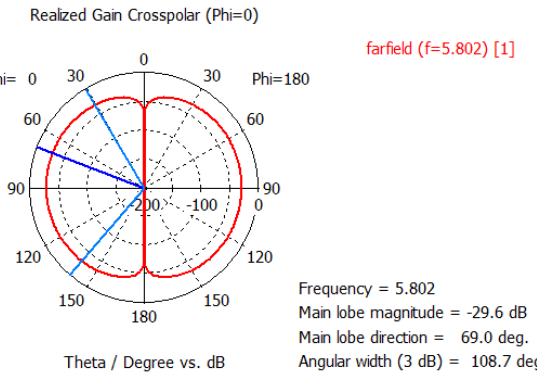

b. Crosspolar

Figure 8. 2D Pattern of the second iteration Minkowski fractal patch antennas when $a_{1}=a_{2}=0.33$.

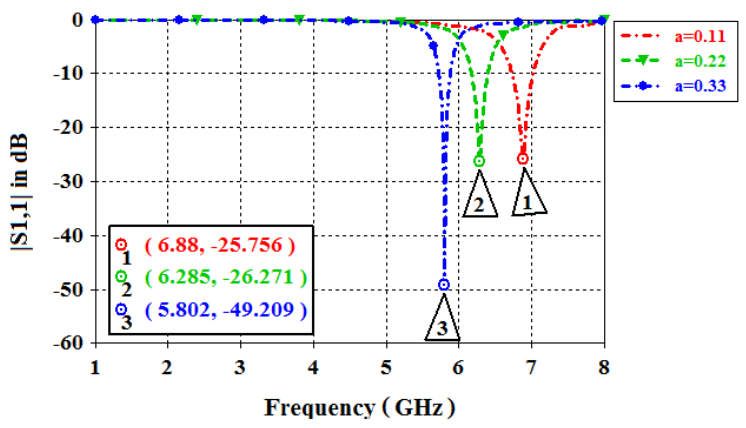

a.

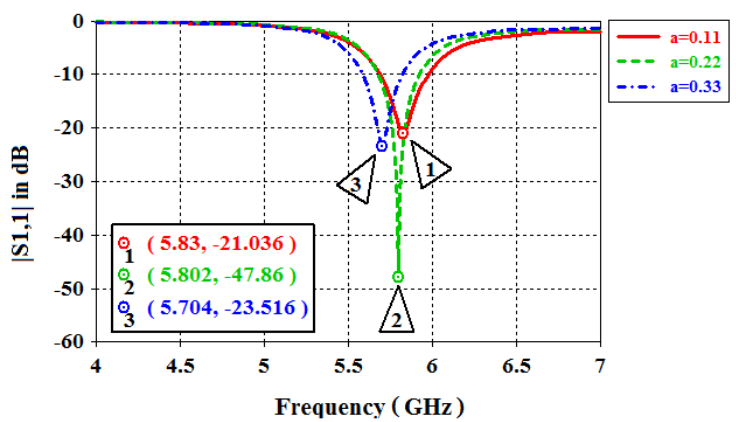

b.

Figure 9. Return loss $S_{11}$ when $a=0.11,0.22$, and 0.33 for the second iteration Minkowski fractal patch antenna (a) $b=$ 0.33 (b) $b=0.22$.

\subsection{Koch Fractal Patch Antenna}

Koch fractal geometry exhibits well-known features that have been used to construct miniaturized monopole and loop antennas [23-25]. By applying the Koch fractal shape to the antennas, the overall electrical length of the antennas increases and the resonance frequency becomes lower than that of conventional monopole, loop, and patch-type antenna. In this paper, Koch fractal geometry is applied to microstrip patch antennas to reduce their overall size. Figure 10 shows four iterations of fractal Koch curves. In this paper, the indentation angle $\alpha$ is taken as a design parameter rather setting it to $60^{\circ}$ as in conventional Koch fractal patch [26]. This gives us an additional design parameter which can be used to achieve extra size reduction (See Fig. 10).

The dimension of the modified Koch fractal patch can be calculated by applying the scales of

$$
\begin{gathered}
S_{2}=S_{3}=\frac{L_{o}}{3} \\
S_{1}=S_{4}=L_{o}\left(\frac{1}{2}-\frac{1}{3} \cos \theta\right) \\
k_{1}=k_{2}=2, h_{1}=3, h_{2}=(3-2 \cos \theta) / 6
\end{gathered}
$$

where $\theta$ is an indentation angle. Substituting these parameters in Eqn. 9, we obtain

$$
2\left(\frac{1}{3}\right)^{D}+2\left(\frac{3-2 \cos \theta}{6}\right)^{D}=1
$$

The solution of this transcendental equation gives the value of $D$ for a given $\theta$.

The perimeter length of the wire $L_{\text {perim }}$ is important parameter that indicates the longer of it is the lowerresonance frequency. The perimeter length of the proposed modified Koch patch geometry, at any angle and at any iteration, is found as

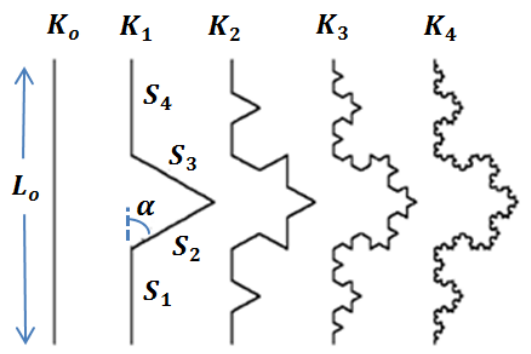

Figure 10. Curves corresponding to the four first iterations of Koch fractal.

$$
L_{\text {perim }}^{(n)}=4\left[\frac{2}{3}\left(\frac{5}{3}-\cos \theta\right)\right]^{n} L_{o}
$$

where $L_{\text {perim }}{ }^{(n)}$ is the perimeter length at iteration $n$ and $L_{o}$ is the one side length of the square patch antenna. The effective area of modified Koch patch at any angle $\theta$ and iteration number $n$ is found to be

$$
A^{(n)}=\left[1-4\left(\sum_{i=1}^{n} \frac{4^{i-1}}{9^{i}}\right) \cos \theta \sin \theta\right] L_{o}^{2}
$$


Table 5 shows the calculated length $L_{\text {perim }}{ }^{(n)}$, area $A^{(n)}$, and dimension $D$ for four iterations and different values of $\theta$.

It is found that as the iteration number and iteration factors increase, the resonance frequencies become lower than those of the zero iteration, which represents a conventional square patch. In other words, microstrip patch antennas employing Koch fractal island geometry can operate at a much lower frequency range while maintaining identical overall antenna characteristics.

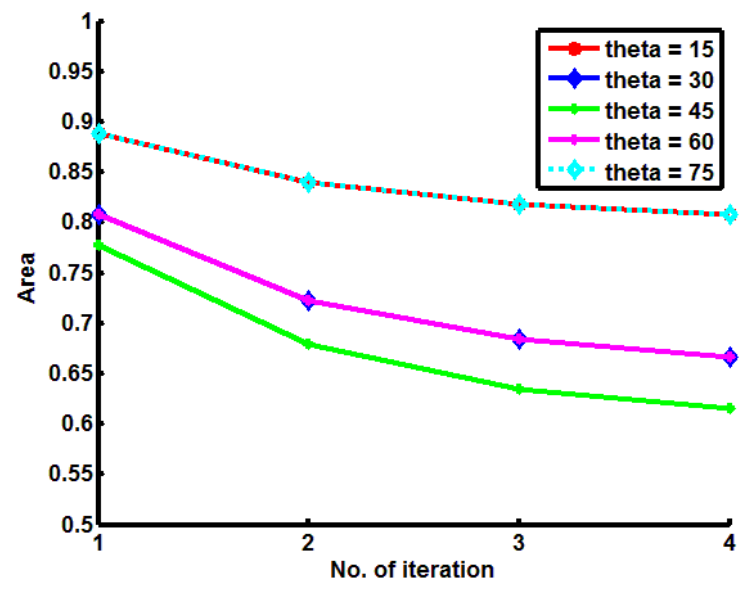

Figure 11. Relation between area of fractal Koch patch antenna with number of iterations for different values of indentation angles.

The enclosed area of the Koch fractal antenna decreases with increaing the number of iterations. The enclosed area is the same at indentation angle $\theta$ and $90^{\circ}-\theta$, this is clear from Fig. 11. Figure 12 indicates that the perimeter length of Koch fractal patch increases with number of iterations. Longer perimeter is obtained when large indentation angle is used. The ratio of perimeter length to enclosed area is large when more iterations and large indentation angle are used as shown in Fig. 13.

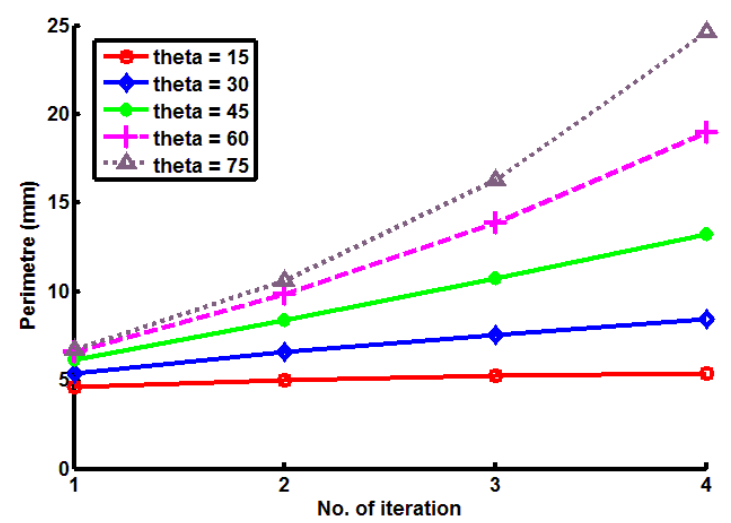

Figure 12. Relation between perimeter of fractal Koch patch antenna for different values of indentation angles and number of iterations.

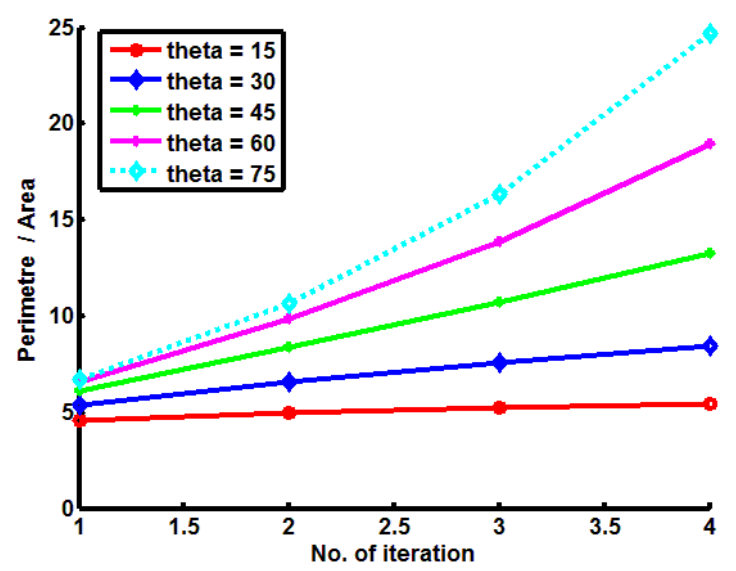

Figure 13. Variation of perimeter to area ratio of fractal Koch patch antenna with indentation angles and number of iterations.

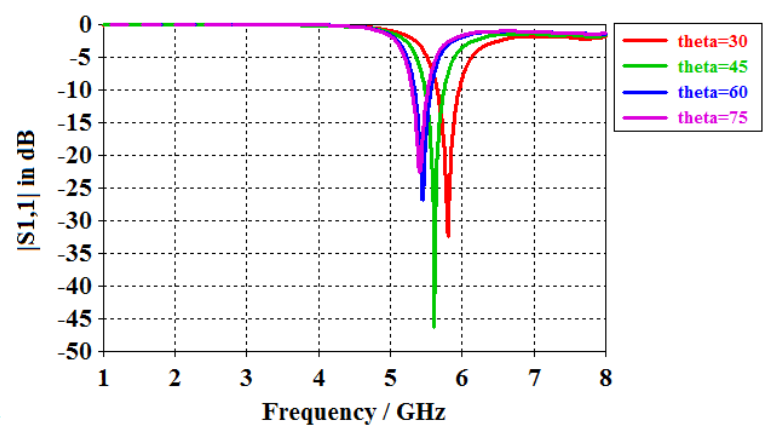

a.

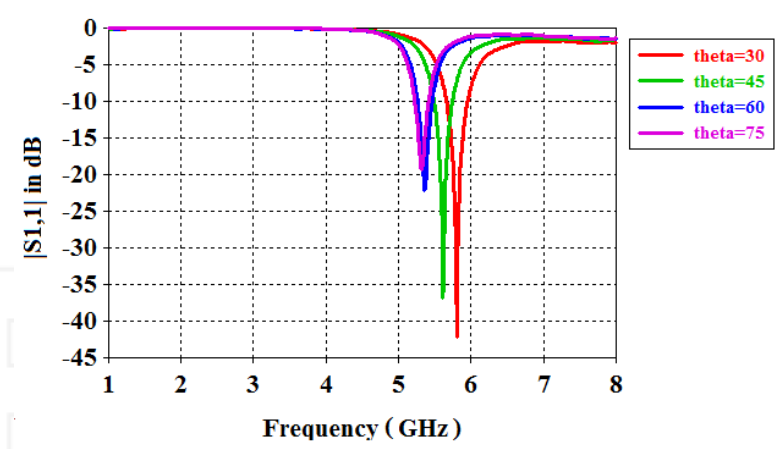

b.

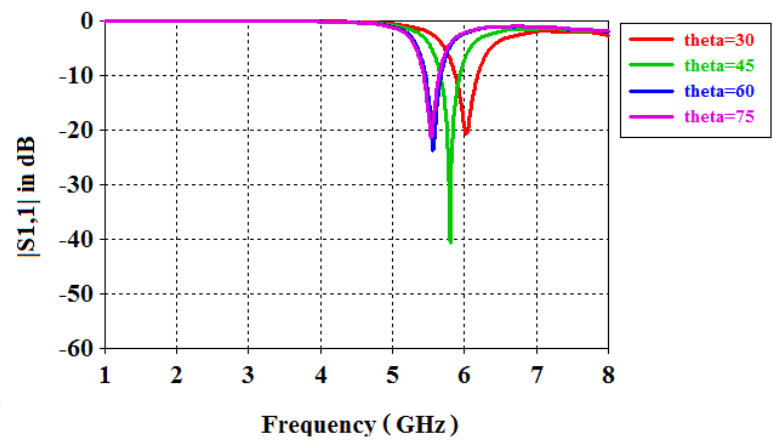

c.

Figure14. Return loss $S_{11}$ for different values of indentation angles $\left(30^{\circ}, 45^{\circ}, 60^{\circ}\right.$, and $\left.75^{\circ}\right)$ for Koch fractal patch antenna. (a) first iteration (b) second iteration (c) third iteration. 
Figure 14 show the variation of the simulated reflection return loss $S_{11}$ with indentation angle for iteration one, two, and three, respectively. The location of $S_{11}$ minimum occurs at lower frequency when indentation angle increases, i.e. the resonance frequency decreases when the indentation angle increases.
The minimum miniaturized size is achieved when third iteration and indentation angle $\theta$ of $75^{\circ}$ are used. This corresponds to $34.06 \%$ reduction in enclose patch area (See Tables 6-8).

\begin{tabular}{cc|cccc|cccc}
\hline \multirow{2}{*}{$\boldsymbol{\theta}(\boldsymbol{d e g})}$. & \multirow{2}{*}{$\boldsymbol{D}$} & \multicolumn{4}{|c|}{$\boldsymbol{L}_{\text {perim }}^{(\boldsymbol{n})}(\boldsymbol{m m})$} & \multicolumn{3}{c}{$\boldsymbol{A}^{(\boldsymbol{n})}\left(\boldsymbol{m m m}^{\mathbf{2}}\right)$} \\
\cline { 3 - 11 } & $\boldsymbol{n = 1}$ & $\boldsymbol{n = 2}$ & $\boldsymbol{n = 3}$ & $\boldsymbol{n}=\mathbf{4}$ & $\boldsymbol{n = 1}$ & $\boldsymbol{n}=\mathbf{2}$ & $\boldsymbol{n}=\mathbf{3}$ & $\boldsymbol{n}=\mathbf{4}$ \\
\hline 15 & 1.017 & 4.09 & 4.18 & 4.28 & 4.38 & 0.889 & 0.840 & 0.818 & 0.808 \\
30 & 1.067 & 4.36 & 5.17 & 5.17 & 5.63 & 0.808 & 0.722 & 0.684 & 0.667 \\
45 & 1.148 & 4.78 & 6.83 & 6.83 & 8.16 & 0.788 & 0.679 & 0.635 & 0.616 \\
60 & 1.261 & 5.33 & 9.48 & 9.48 & 12.6 & 0.808 & 0.722 & 0.684 & 0.667 \\
75 & 1.411 & 5.98 & 8.93 & 13.3 & 19.9 & 0.889 & 0.840 & 0.818 & 0.808 \\
\hline
\end{tabular}

Table 5. Variation of $D, L_{\text {perim }}{ }^{(n)}$, and $A^{(n)}$ as a function of nand $\theta$ for the modified Koch fractal patch antenna.

\begin{tabular}{|c|c|c|c|c|c|}
\hline$\theta$ (deg.) & 0 & 30.00 & 45.00 & 60.00 & 75.00 \\
\hline Dimension & 1.000 & 1.067 & 1.148 & 1.261 & 1.411 \\
\hline$\Delta \boldsymbol{A}(\%)$ & - & 20.79 & 25.00 & 29.94 & 31.11 \\
\hline$L_{\text {perim }}(\mathrm{mm})$ & 40.00 & 38.78 & 41.40 & 44.64 & 49.60 \\
\hline$A\left(m m^{2}\right)$ & 100.00 & 63.96 & 58.33 & 56.57 & 61.23 \\
\hline$L_{\text {perim }} / A$ & 0.40 & 0.60 & 0.71 & 0.79 & 0.81 \\
\hline$S_{11}(d B)$ & -33.20 & -33.82 & -34.48 & -31.25 & -41.87 \\
\hline$B W(M H z)$ & 356 & 304 & 276 & 274 & 274 \\
\hline Gain $(\mathrm{dB})$ & 4.04 & 3.62 & 3.45 & 3.41 & 3.43 \\
\hline
\end{tabular}

Table 6. Simulated parameters of the first iteration Koch fractal patch antenna.

\begin{tabular}{|c|c|c|c|c|c|}
\hline$\theta$ (deg. $)$ & 0 & 30.00 & 45.00 & 60.00 & 75.00 \\
\hline D & 1.000 & 1.067 & 1.148 & 1.261 & 1.411 \\
\hline$\Delta \boldsymbol{A}(\%)$ & - & 17.55 & 21.50 & 31.11 & 31.77 \\
\hline$L_{\text {perim }}(\mathrm{mm})$ & 40.00 & 43.09 & 50.63 & 59.02 & 73.75 \\
\hline$A\left(\boldsymbol{m m}^{2}\right)$ & 100.00 & 59.52 & 55.30 & 49.47 & 57.27 \\
\hline$L_{\text {perim }} / A$ & 0.40 & 0.72 & 0.95 & 1.18 & 1.28 \\
\hline$S_{11}(d B)$ & -33.20 & -42.18 & -39.88 & -37.91 & -37.05 \\
\hline$B W(M H z)$ & 356 & 292 & 268 & 256 & 257 \\
\hline Gain (dB) & 4.04 & 3.57 & 3.44 & 3.32 & 3.36 \\
\hline
\end{tabular}

Table 7. Simulated parameters of the second iteration Koch fractal patch antenna. 


\begin{tabular}{|c|c|c|c|c|c|}
\hline & & & & 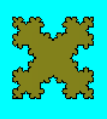 & 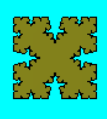 \\
\hline$\theta$ (deg. $)$ & 0 & 30 & 45 & 60 & 75 \\
\hline Dimension & 1.000 & 1.067 & 1.148 & 1.261 & 1.411 \\
\hline$\Delta \boldsymbol{A}(\%)$ & - & 22.56 & 32.35 & 31.77 & 34.06 \\
\hline$L_{\text {perim }}(\mathrm{mm})$ & 40.00 & 45.50 & 56.18 & 78.31 & 108.33 \\
\hline$A\left(\boldsymbol{m} m^{2}\right)$ & 100.00 & 52.97 & 42.97 & 46.68 & 53.90 \\
\hline$L_{\text {perim }} / A$ & 0.40 & 0.86 & 1.30 & 1.68 & 2.00 \\
\hline$S_{11}(d B)$ & -33.20 & -30.10 & -27.31 & -37.32 & -35.41 \\
\hline$B W(M H z)$ & 356 & 289 & 275 & 242 & 255 \\
\hline Gain (dB) & 4.04 & 3.42 & 3.32 & 3.25 & 3.29 \\
\hline
\end{tabular}

Table 8. Simulated parameters of the third iteration Koch fractal patch antenna.

\section{Modified Fractal Antennas}

This section introduces modified Minkowski fractal antenna, Sierpinski Gasket fractal as a bowtie shape patch antenna, and hybrid fractal as modified Koch fractal ring patch with and without repeated scaled-Koch ring fractal patch antenna. The concepts of modification are identified and supported by simulation results.

\subsection{Modified Minkowski Fractal Antenna}

In order to achieve a reduced size of the patch antenna, a new geometry is proposed here which is derived from fractal Minkowski patch geometry. The recursive iteration of this fractal starts from the zero-order normal patch then two scales are used to generate two subpatches, one with scale $s e_{1}$, and the one other with a scalesc $c_{1}$. The $s e_{1}$ scaled patch at the centre and sc1 scales the patch at the four corners of $s e 1$ scaled centered patch. After succeed iteration new scaless $s e_{2}$, and $s c_{2}$ are used, and so on, as shown in Fig. 15.

The modified Minkowski fractal antenna (MMPFA) for five patch shapes and different scales are simulated. The simulated results, return $\operatorname{loss} S_{11}$, gain, and bandwidth are listed in Table 9. We notice that all shapes of the proposed antenna (MMPFA1 to MMPFA6) except MMPFA4 have more reduction in size than other fractal shapes mentioned in this paper (See Table 9).

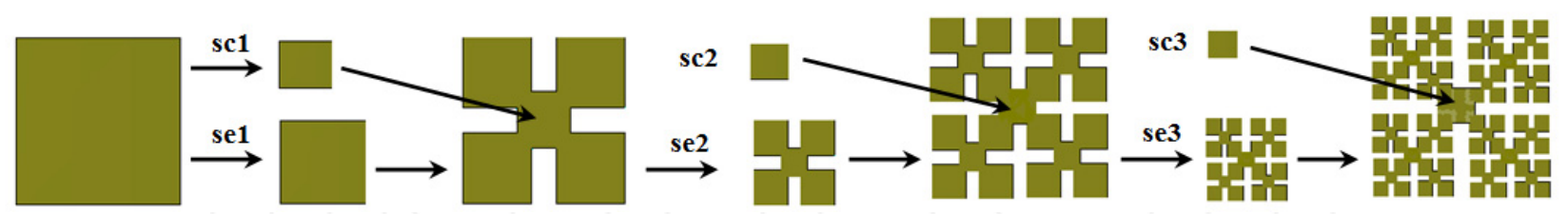

Figure 15. Iteration of modified Minkowski fractal patch antenna.

\begin{tabular}{lcccccc}
\hline & MMFA1 & MMFA2 & MMFA3 & MMFA4 & MMFA5 & MMFA6 \\
& & & & \\
& & & & \\
& & &
\end{tabular}

Table 9. Simulated parameters of the proposed modified Minkowski fractal patch antenna. 


\begin{tabular}{|c|c|c|c|c|c|}
\hline & Zero Iteration & First Iteration & Second Iteration & Third Iteration & Fourth Iteration \\
\hline$\Delta \boldsymbol{A}(\%)$ & - & 48.48 & 49.16 & 50.01 & 52.39 \\
\hline$S_{11}(d B)$ & -33.20 & -41.29 & -34.38 & -44.23 & -31.77 \\
\hline$B W(M H z)$ & 356 & 172 & 164 & 166 & 178 \\
\hline Gain (dB) & 4.04 & 2.23 & 2.11 & 2.03 & 2.23 \\
\hline
\end{tabular}

Table 10. Simulated parameters of bowtie Sierpinski fractal antenna.

The 2D copolar and cross polar patterns at $5.8 \mathrm{GHz}$ are introduced in Fig. 16. We one can notice from Fig. 16 that these patterns are similar to the patterns of the reference patch antenna A simulation of the return loss $S_{11}$ of MMFA6 that has the highest reduction in size area reduction (59.80\%) is shown in Fig. 17.

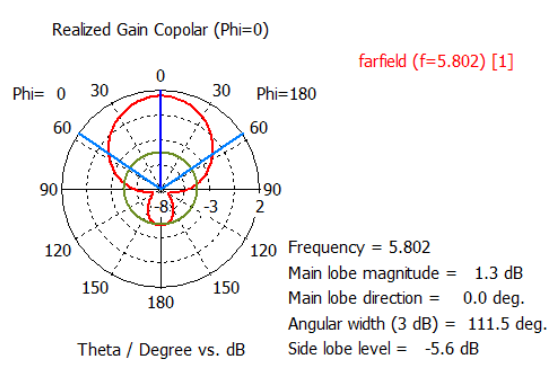

a.

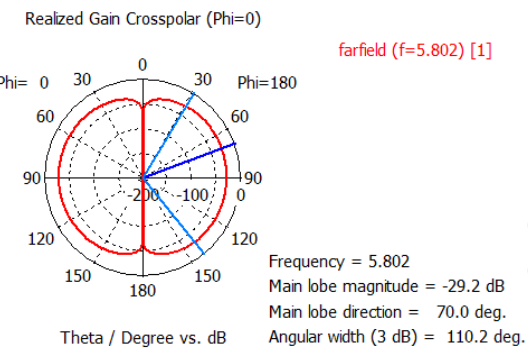

b.

Figure 16. 2D Pattern of modified Minkowski fractal patch (MMFA6).

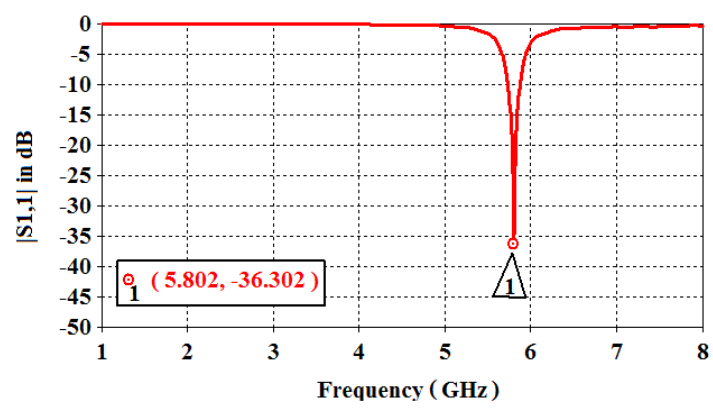

Figure 17.Simulated return loss $S_{11}$ of the MMFA6 proposed modified Minkowski fractal patch antenna.

\subsection{Bowtie Sierpinski Gasket Fractal Antenna}

The Sierpinski fractal patch antenna is one of widely used and studied by literature because of its behaviour and its multiband characteristics. In this paper a two Sierpinski fractal patches (as a bowtie shape) are used as the unit cell for the fractal geometry.

Four iterations of bowtie Sierpinski fractal patch antenna are simulated and the results at resonant frequency of $5.8 \mathrm{GHz}$ are listed in Table 10. The return loss $S_{11}$, gain and bandwidth are the main results that tabulated in Table 10. Note that the four iterations give approximately the same size reduction. With a fouriteration antenna, a reduction $\Delta A$ of $52.39 \%$ is obtained

\section{Hybrid Modified Koch Fractal Antenna}

A new hybrid fractal patch antenna is proposed to miniaturize the size of patch antenna compared with Koch fractal antenna mentioned in section III that suitable for RFID. The fractal iteration starts from a square patch subtracted from $45^{\circ}$ rotated half size reference square patch as a zero order then a fractal Koch is applied to the inner of the zero-order reference patch. Two iterations and four indentation angles are simulated for single ring patch, and then a second iteration for double ring with a half is simulated too.

Tables 11 to 13 show the results of the proposed hybrid fractal antenna. These tables present the return loss $S_{11}$, gain and bandwidth beside the reduction in patch size.

Note that more reduction in area is obtained for this patch antenna compared with previously Koch fractal patch type. A maximum of $42 \%$ reduction in $A$ for $\theta=75^{\circ}$ indentation angle for double Koch second iteration fractal patch antenna (see Table 13) compared to maximum reduction in $\mathrm{A}$ of $34 \%$ obtained when $\theta=75^{\circ}$ for the third iteration Koch fractal patch antenna ( see Table 8). 


\begin{tabular}{|c|c|c|c|c|c|}
\hline$\theta$ (deg. $)$ & - & 30 & 45 & 60 & 75 \\
\hline$\Delta \boldsymbol{A}(\%)$ & - & 30.86 & 32.10 & 34.71 & 37.27 \\
\hline$S_{11}(d B)$ & -33.20 & -31.44 & -36.35 & -31.94 & -36.54 \\
\hline$B W(M H z)$ & 356 & 264 & 264 & 258 & 271 \\
\hline Gain (dB) & 4.04 & 3.32 & 3.29 & 3.21 & 3.26 \\
\hline
\end{tabular}

Table 11. Simulated parameters of the first iteration single modified Koch fractal patch antenna.

\begin{tabular}{|c|c|c|c|c|c|}
\hline $\boldsymbol{\theta}$ (deg. $)$ & - & 30 & 45 & 60 & 75 \\
\hline$\Delta \boldsymbol{A}(\%)$ & - & 34.06 & 35.35 & 38.38 & 39.16 \\
\hline$S_{11}(d B)$ & -33.20 & -46.51 & -49.27 & -42.27 & -30.53 \\
\hline$B W(M H z)$ & 356 & 273 & 259 & 259 & 274 \\
\hline Gain (dB) & 4.04 & 3.26 & 3.19 & 3.2 & 3.24 \\
\hline
\end{tabular}

Table 12. Simulated parameters of the second iteration single modified Koch fractal patch antenna.

\begin{tabular}{|c|c|c|c|c|c|}
\hline$\theta($ deg. $)$ & - & 30 & 45 & 60 & 75 \\
\hline$\Delta A(\%)$ & - & 39.86 & 41.48 & 41.48 & 42.24 \\
\hline$S_{11}(d B)$ & -33.20 & -30.34 & -36.42 & -23.59 & -28.16 \\
\hline$B W(M H z)$ & 356 & 274 & 275 & 247 & 259 \\
\hline Gain (dB) & 4.04 & 3.29 & 3.26 & 3.18 & 3.19 \\
\hline
\end{tabular}

Table 13. Simulated parameters of the second iteration double modified Koch fractal patch antenna.

Figure 18 show the relation between return loss $S_{11}$ and indentation angle for iteration one, two and three of hybrid Koch fractal patch antenna. Again as stated previously in Koch fractal antenna, lower resonance frequency is achieved for large indentation angle. 


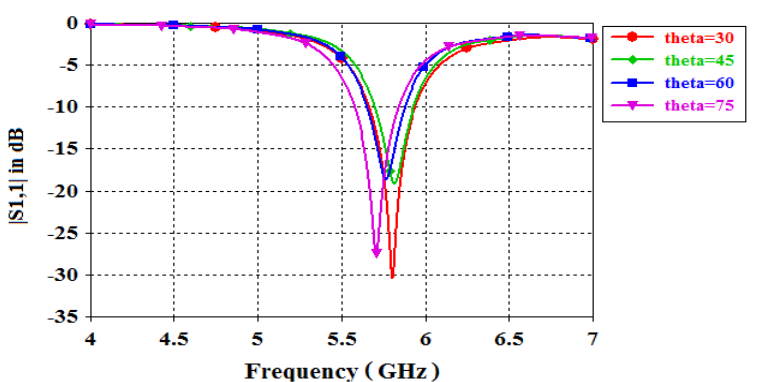

a.

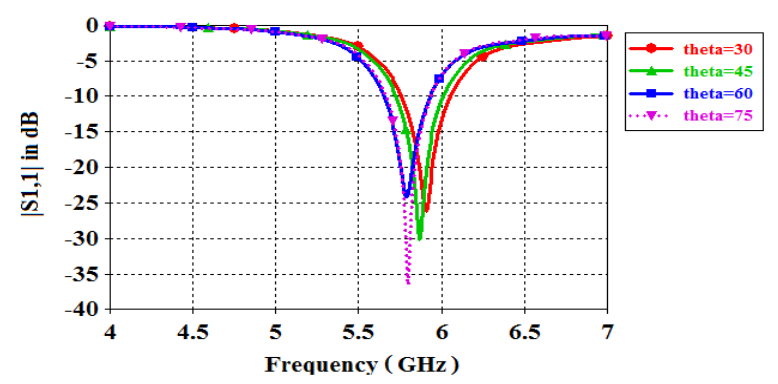

b.

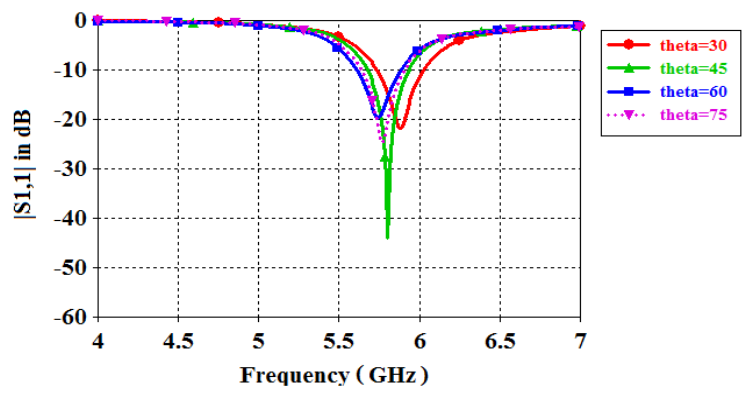

c.

Figure18. Return loss $\mathrm{S}_{11}$ for different values of indentation angles $\left(30^{\circ}, 45^{\circ}, 60^{\circ}\right.$, and $\left.75^{\circ}\right)$ for hybrid modified Koch fractal patch antenna. (a) single first iteration (b) single second iteration (c) double second iteration.

6. Relation Comparison of Different Types of Fractal Patches Antenna

Table 14 summarises the results of the above analysis. This table indicates that modified Minkowski fractal patch antenna gives the best reduction in patch area which is $59.80 \%$.

\begin{tabular}{|c|c|}
\hline Antenna Type & Reduction in A (\%) \\
\hline 1. Modified Minkowski & 59.80 \\
\hline $\begin{array}{l}\text { 2. } \begin{array}{l}\text { Bowtie Sierpinski } \\
\text { gasket }\end{array} \\
\end{array}$ & 52.39 \\
\hline 4. Fractal Minkowski & 49.02 \\
\hline 5. Hybrid Koch & 42.24 \\
\hline 5. Koch & 34.06 \\
\hline 6. Sierpinski carpet & 20.79 \\
\hline
\end{tabular}

Table 14. Reduction in enclosed patch area for different fractal patch antennas.

\section{Conclusions}

Various fractal patch antenna based on aperture coupled feeding have been designed and simulated by CST MWS 2010. New design concepts have been deduced from extensive study of these fractal shapes patch antennas. The ratio of perimeter length of patch to its enclosed area is a main factor for reduction of a given patch geometry. The large of this ratio the more reduction in the size is obtained.

A modified fractal Minkowski fractal patch antenna has been proposed that give most reduction in size among other fractal geometries. Also a hybrid Koch fractal has been introduced to miniaturize the patch antenna.

\section{References}

[1] K. Finkenzeller, "RFID Handbook Fundamentals and Applications in Contactless Smart Cards, Radio Frequency Identification and Near-Field Communication", 3rd Ed. New York; Wiley, 2010.

[2] K-T. Kim, J.-H. Ko, K. Cho, and H.-S. Kim, "Robust Optimum Design of PIFA for RFID Mobile Dongle Applications", IEEE Transactions on Magnetics, Vol. 47, No. 5, May 2011, pp. 962-965.

[3] A. T. Mobashsher, M. T. Islam, and N. Misran, "A Novel High-Gain Dual-Band Antenna for RFID Reader Applications", IEEE Antennas and Wireless Propag. Letts., Vol. 9, 653-655, Sep. 2010.

[4] V. P. Plessky, L. M. Reindl "Review on SAW RFID Tags," IEEE Transactions on Ultrasonics, Ferroelectrics, and Frequency Control, vol. 57, no. 3, March 2010, pp. 654-668.

[5] F. Alimenti, M. Virili, G. Orecchini, P. Mezzanotte, V. Palazzari, M. M. Tentzeris, and L. Roselli, “A New Contactless Assembly Method for Paper Substrate Antennas and UHF RFID Chips," IEEE Transactions ON Microwave Theory and Techniques, Vol. 59, No. 3, March 2011, pp. 627-637.

[6] P.J. Soh, G. A. E. Vandenbosch, S.L. Ooi and M.R.N. Husna, "Wearable dual-band Sierpinski fractal PIFA using conductive fabric," Electronics Letters 17th March 2011 Vol. 47 No. 6.

[7] A. Mehdipour, I. D. Rosca, A.-R. Sebak, C. W. Trueman, and S. V. Hoa, "Full-Composite Fractal Antenna Using Carbon Nanotubes for Multiband Wireless Applications," IEEE Antennas and Wireless Propagation Letters, Vol. 9, 2010, pp. 891-894.

[8] J. Barral, S. Seuret, "Recent Developments in Fractals and Related Fields", Springer, New York, 2010.

[9] D. H. Werner, and S. Ganguly, "An overview of fractal antenna engineering research," IEEE Antennas and Propagation Magazine, Vol. 45, 38\{57, Feb. 2003. 
[10] J. P. Gianvittorio, and Y. R. Samii, “Fractal antennas: A novel antenna miniaturization technique and applications," IEEE Antennas and Propagation Magazine, Vol. 44, No. 1, Feb. 2002.

[11] K. J. Falconer, Fractal Geometry: Mathematical Foundations and Applications, John Wiley \& Sons, New York, $3^{\text {rd }}$ ed., 2003.

[12] P. L. Sullivian and D. H. Schaubert, "Analysis of an Aperture Coupled Microstrip Antenna," IEEE Transactions on Antenna propagation, Vol. AP-34, 1986, pp. 977-984.

[13] M. A. Saed, "Efficient Method for Analysis and Design of Aperture Coupled Rectangular Microstrip Antenna," IEEE Transactions on Antenna Propagation, Vol. AP-41, 1993, pp. 986-988.

[14] F.-Y. Kuo, P.-H. Pan, C.-Y. Chiang, H.-T. Hsu, and H.-T. Chou, "Dual-band Aperture-Coupled Patch Antenna for RFID Mobile Terminal Applications," Proceedings of Asia-Pacific Microwave Conference 2010.

[15] S. N. Sinha, and M. Jain, "A Self- Affine Fractal Multiband Antenna," IEEE Antennas and Wireless Propag. Letts., Vol. 6, pp. 110-112, 2007.

[16] H. Yang, S. Yan, L. Chen, and H. Shi, "Investigation and Design of a Modified Aperture-Coupled Fractal Antenna for RFID Applications," ISECS International Colloquium on Computing, Communication, Control, and Management, 2008, pp. 505-509.

[17] C. A. Balanis, Antenna Theory: Analysis and Design, $3^{\text {rd }}$ ed. Hoboken, NJ: Wiley, 2005.

[18] R. Ghatak, R. K. Mishra, and D. R. Poddar, "Perturbed Sierpinski Carpet Antenna with CPW Feed for IEEE $802.11 \mathrm{a} / \mathrm{b}$ WLAN Application", IEEE Antennas and Wireless Propagation Letters, Vol. 7, 2008, pp. 742-744.
[19] H. Oraizi and S. Hedayati, "Miniaturized UWB Monopole Microstrip Antenna Design by the Combination of Giusepe Peano and Sierpinski Carpet Fractals," IEEE Antennas and Wireless Propagation Letters, Vol. 10, 2011, pp. 67-70.

[20] M. Comisso, "Theoretical and numerical analysis of the resonant behaviour of the Minkowski fractal dipole antenna," IET Microw. Antennas Propag., 2009, Vol. 3, Iss. 3, pp. 456-464

[21] M. Rusu, M. Hirvonen, H. Rahimi, P. Enoksson, C. Rusu, N. Pesonen, O. Vermesan, and H. Rustad, "Minkowski Fractal Microstrip Antenna for RFID Tags," Proceedings of the 38th European Microwave Conference 2008.

[22] K.J. Vinoy, and A. Pal, "Dual-frequency characteristics of Minkowski-square ring antennas," IET Microw. Antennas Propag., 2010, Vol. 4, Iss. 2, pp. 219-224.

[23] K. J. Vinoy, J. K. Abraham, and V. K. Varadan, "On the Relationship Between Fractal Dimension and the Performance of Multi-Resonant Dipole Antennas Using Koch Curves," IEEE Transactions On Antennas and Propagation, Vol. 51, No. 9, pp. 2296-2303, Sep.2003.

[24] K.Q. da Costa and V. Dmitriev, "Theoretical analysis of a modified Koch monopole with reduced dimensions," IEE Proc.-Microw. Antennas Propag., Vol. 153, No. 5, October 2006.

[25] W. J. Krzysztofik, "Modified Sierpinski Fractal Monopole for ISM-Bands Handset Applications", IEEE Transactions on Antennas and Propagation, Vol. 57, No. 3, March 2009.

[26] C. P.-Baliarda, J. Romeu, R. Pous, and A. Cardama, "On the Behavior of the Sierpinski Multiband Fractal Antenna", IEEE Transactions on Antennas and Propagation, Vol. 46, No. 4, pp. 517-5, 24, April 1998. 